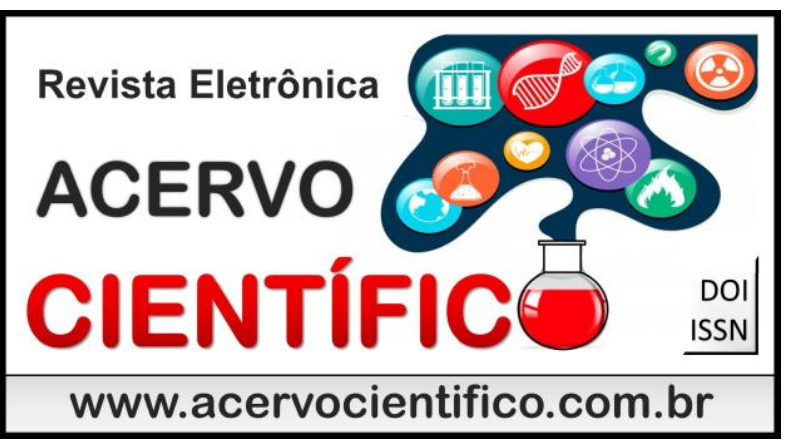

RELATO DE CASO

Recebido em: $3 / 2019$

Aceito em: 4/2019

Publicado em: 8/2019

\title{
Epilepsia abdominal: relato de caso
}

\author{
Abdominal epilepsy: case report
}

\section{Epilepsia abdominal: relato de caso}

Natasha Nascimento Paiva ${ }^{1 *}$, Roberta Alves de Macedo ${ }^{1}$, Taís Teixeira Chibini ${ }^{1}$, Thaís Martins Oliveira ${ }^{1}$.

Resumo: Este relato de caso tem como objetivo reconhecer precocemente o diagnóstico para tratamento adequado e evitar possíveis complicações da epilepsia abdominal. No presente estudo, a paciente apresenta dor abdominal difusa associado à queda do estado geral e sonolência intensa durante os episódios. Após iniciar o uso contínuo de anticonvulsivantes, houve melhora do quadro clinico. A epilepsia abdominal é uma patologia com alto nível de complexidade e relativamente rara, com características de manifestações abdominais acompanhadas ou não a outros sintomas e, por ser raro, é de difícil diagnóstico.

Palavras chave: Dor abdominal, Epilepsia abdominal, Criança.

Abstract: This case report aims to recognize early diagnosis for appropriate treatment and avoid possible complications by abdominal epilepsy. In the present study, the patient presented diffuse abdominal pain associated with general state decrease and intense drowsiness during the episodes. After starting continuous use of anticonvulsants, there was improvement in clinical picture. Abdominal epilepsy is a pathology with high level of complexity and rarely, with characteristics of abdominal manifestations accompanied or not by other symptoms and, because it is rare, it is difficult to diagnose.

Key words: Abdominal pain, Abdominal epilepsy, Child.

Resumen: Este relato de caso tiene como objetivo reconocer precozmente el diagnóstico para tratamiento adecuado y evitar posibles complicaciones de la epilepsia abdominal. En el presente estudio, la paciente presenta dolor abdominal difuso asociado a la caída del estado general y somnolencia intensa durante los episodios. Después de iniciar el uso continuo de anticonvulsivantes, hubo mejoría del cuadro clínico. La epilepsia abdominal es una patología con alto nivel de complejidad y relativamente rara, con características de manifestaciones abdominales acompañadas o no a otros síntomas y, por ser raro, es de difícil diagnóstico.

Palabras clave: Dolor abdominal, Epilepsia abdominal, Niño.

${ }^{1}$ Universidade de Franca (UNIFRAN), Franca, São Paulo, Brasil.*E-mail: natasha nascimento@hotmail.com 


\section{INTRODUÇÃO}

A Epilepsia Abdominal (EA) pode ser definida como uma associação entre alteração do nível e consciência e episódios paroxísticos com suas manifestações clinicas, além de anormalidades eletroencefalográficas e bom comportamento frente à terapia anticonvulsivante, porém se mostra de difícil diagnóstico devido a alterações neurológicas de alta amplitude e de dor abdominal recorrente na infância (RIBEIRO RM et al, 1964).

A dor abdominal caracterizada como recorrente tem um conceito que afirma a presença de três ou mais ocorrências de dor na região do abdômen superior a três meses. As dores abdominais recorrentes são consideradas dentro deste conceito se atrapalham a atividade cotidiana do indivíduo, elas podem aparecer como episódios isolados paroxísticos de dor Peri umbilical, associado também a alguma disfunção do tudo digestivo e com dispepsia e presença de dor abdominal (BOYLE JT et al, 1997).

O quadro clinico da do abdominal por ser bastante amplo pode gerar dúvidas se não associado a outras manifestações abdominais, e provavelmente a epilepsia abdominal não será a primeira escolha para um diagnóstico por ser bastante rara. Embora os sintomas abdominais possam ser semelhantes aos da síndrome do intestino irritável, a epilepsia abdominal pode ser distinguida da última condição pela presença de consciência alterada durante alguns dos episódios, uma tendência ao cansaço após um episódio e um EEG anormal (ZARLING EJ,1984).

Duas décadas atrás, houve tentativa de elaborar critérios para a definição desta entidade, os quais são: dor abdominal paroxística, exclusão de dor abdominal visceral patológica, alteração do estado mental durante pelo menos alguns episódios, EEG anormal e resposta positiva aos anticonvulsivantes. Sua classificação é parte de um grupo maior (epilepsias parciais) e não requer uma classificação especial porque a dor abdominal está geralmente associada a outras manifestações ictais compatível com o diagnóstico de crises parciais (FRANZON RC et al, 2002).

O diagnóstico precoce da doença é pouco frequente pelas dificuldades já relatadas. O quadro clínico é amplo e geralmente caracterizado por desconforto abdominal, náuseas associadas a convulsões, crises de ausência, alteração do nível de consciência após o início da crise. Em 1941, foi demonstrada a origem cerebral dessas manifestações abdominais que possuem direta relação diencefálica, o diencéfalo é uma estrutura ímpar que só é vista na porção mais inferior de cérebro. (RIBEIRO RM et al, 1964).

É possível realizar o diagnóstico de EA pelo quadro clínico com a predominância de sintomas paroxístico abdominais e distúrbios da consciência e principalmente pela resposta favorável a terapêutica anticonvulsivante e alterações encefalograficas, bastante frequentes nesses casos porém não exclusivas (SOLANA LJ, 1994).

Desta maneira, o objetivo deste relato de caso é reconhecer precocemente a clínica para posterior diagnóstico e tratamento adequado e evitar possíveis complicações da epilepsia abdominal.

\section{RELATO DE CASO}

O presente relato foi feito com o intuito de analisar as manifestações clínicas e eletrencefalográficas de uma criança portadora de epilepsia abdominal. O objetivo é mostrar o valor da eletrencefalografia na investigação diagnóstica dessas síndromes abdominais de características paroxísticas no público pueril, principalmente pela alta gama de patologias que podem se manifestar através de dor abdominal que não pode ser especificada. Este relato de caso analisa todo contexto da epilepsia abdominal apesar de ser um diagnóstico de exclusão.

O trabalho foi desenvolvido através da análise de um caso clínico de um paciente oriundo de uma clínica pediátrica localizada em uma cidade do interior estado de São Paulo que, mediante a assinatura do termo de consentimento livre esclarecido, seu prontuário foi revisado através da literatura relacionada ao tema. Foi analisado todo o contexto da epilepsia abdominal pediátrica, verificando a epidemiologia (em estágios pueris) 
predominância, o quadro clinico, o diagnóstico e as condutas terapêuticas (usados em nossa paciente a oxicaramazepina), analisando as mesmas quanto a diagnósticos diferenciais e complicações.

Paciente RSMS, sexo feminino, 8 anos, chegou a Cuidar Clínica Pediátrica com queixa de dor abdominal em queimação, de localização difusa, 10/10 na escala visual de dor, sem irradiação, sem fatores de melhora ou de piora, queda do estado geral e sonolência intensa. Durante esses episódios, não houve vômitos, sialorreia, liberação esfincteriana e espamos musculares. Negou febre, perda de peso, náusea e vômitos e sintomas urinários, cardiovascular, respiratório e neurológicos.

Referiu fezes Bristol 2, com hábito de duas vezes na semana associado a dificuldade para evacuação, sem outras alterações. Genitora referiu que durante o pré-natal e parto cesárea não houve intercorrência, desenvolvimento neuropsicomotor compatível com a idade e sexo, vacinação em dia. Nega alergias e uso de medicamentos contínuo e internações prévias. Hábito alimentar pouco diversificado, pobre em frutas, verduras, legumes e alimentos contendo fibras. Ingesta hídrica de um litro e meio de água diário e pratica atividade física uma vez por semana na escola. Sem dados relevantes no histórico familiar.

No exame físico apresentou mal estado geral, corada, hidratada, acianótica, anictérica, eupneica, normocardica, hipoativa, pele com turgor e elasticidade preservadas, panículo adiposo bem distribuído e homogêneo, musculatura eutônica e eutrófica, esqueleto harmônico compatível com idade e sexo, lúcida, memória remota e recente, normocardia, 2 bulhas ritimicas normofoneticas sem presença de sopro, murmúrio vesicular presente sem presença de ruídos adventícios, abdômen plano, ausência de lesões, circulação colateral e herniações, ruídos hidroaéreos presentes difusamente, sem alterações à percussão e ausência de visceromegalias, Dor a palpação superficial e profunda em todo o abdômen e ausência de resposta à descompressão brusca. Escala de Tanner em M0 e P0.

Nesse primeiro atendimento foi tratada para quadro de gastrite e refluxo com omeprazol, cloridrado de ranitidina e domperidona, não havendo nenhuma melhora do quadro.

Após 2 dias, a paciente retornou ao pronto-socorro com o exame físico igual à primeira consulta, sendo internada por 3 dias. No tratamento, foram prescritos os mesmos medicamentos da consulta anterior, com melhora discreta da dor abdominal. Foram solicitados ultrassonografia de abdômen total e raio-x de tórax (conforme figura 1), sendo ambos sem alterações.

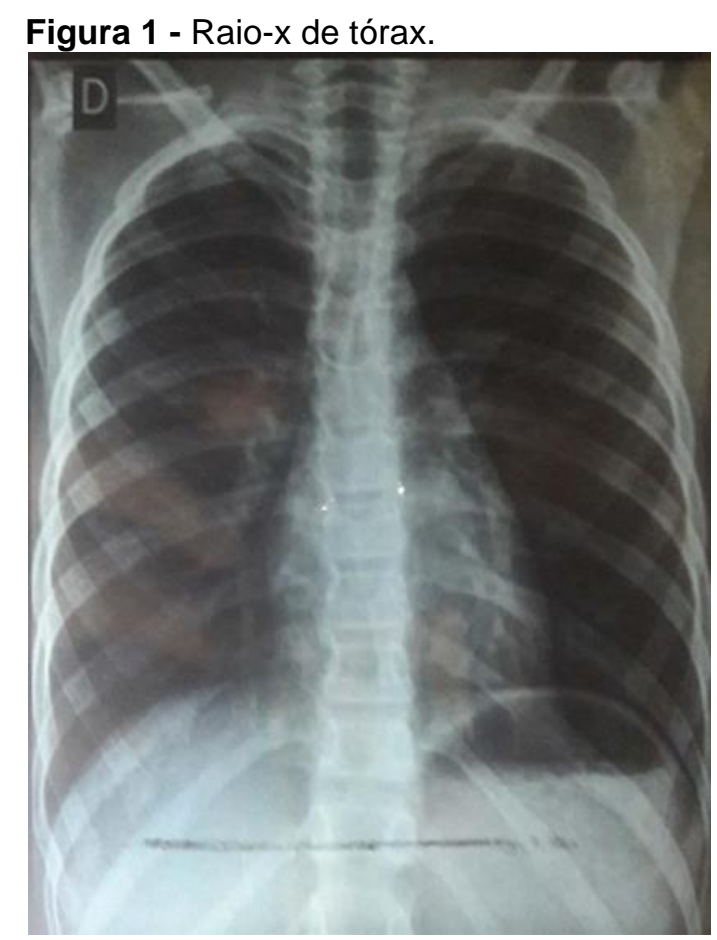

Fonte: Autores, 2019. 
Um dia após alta hospitalar, por persistência do quadro, a progenitora levou novamente a paciente em consultório pediátrico que solicitou parecer do gastroenterologista e da neurologia. Foram solicitados pela gastroenterologista exames laboratoriais para excluir processos alérgicos (Tabela 1) e a neurologia solicitou: eletroencefalograma (ECG - Figura 2) e tomografia de crânio, todos sem alterações. Como os diagnósticos diferenciais foram excluídos a partir dos exames solicitados foi então prescrito oxcarbazepina e desde então a paciente permaneceu assintomática.

Figura 2 - Eletroencefalograma em vigília, dentro dos limites da normalidade.

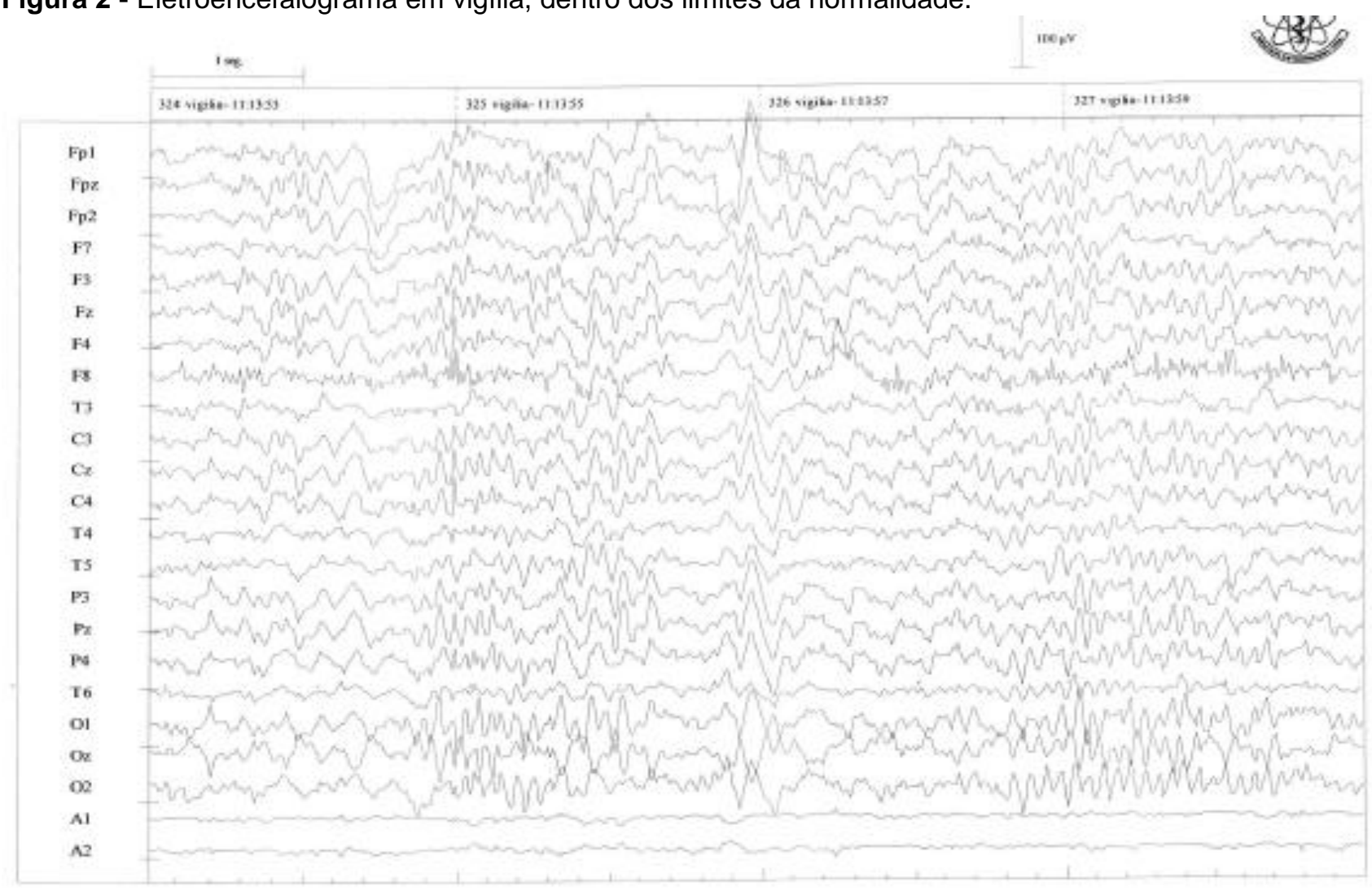

Fonte: Autores, 2019.

Tabela 1 - Exames laboratoriais.

\begin{tabular}{ll}
\hline Indicadores & Resultado \\
\hline $\mathrm{Hb}$ & $13 \mathrm{~g} / \mathrm{dL}$ \\
$\mathrm{Ht}$ & $38 \%$ \\
Leucócitos & $10.000 \mathrm{~mm}^{3}$ \\
Plaquetas & $352.000 \mathrm{~mm}^{3}$ \\
Ferro sérico & $103 \mathrm{mcg} / \mathrm{dL}$ \\
IgE específico para cochicina & Negativo \\
IgE específico para carne de vaca & Negativo \\
IgE específico para tartrazina & Negativo \\
IgG específico para gema de ovo & Negativo \\
IgE específica para Alfa-Lactobulina & Negativo \\
IgE específico para caseína & Negativo \\
IgE específico para glúten & Negativo \\
Anti- gliadina (IgG glúten) & Negativo \\
Anti- gliadina (IgA glúten) & Negativo \\
\hline
\end{tabular}

Fonte: Autores, 2019. 


\section{DISCUSSÃO}

A epilepsia abdominal (EA) é umas das causas mais raras de dor abdominal, sendo caracterizada em uma miscelânea de sintomas convergidos em sinais pertinentes paroxísticos como desconforto abdominal, náuseas, crises convulsivas, inquietude, alteração da consciência, período pós ictal e angústia pueril (SOLANA LJ, 1994). O quadro clínico comparando 21 crianças mostra a versatilidade da enfermidade à medida que houve a apresentação dos sintomas associada com crises convulsivas psicomotoras e alteração de consciência (RIBEIRO RM et al, 1964). Neste caso relatado, a paciente apresentava momentos de sonolência exacerbado e alteração do nível de consciência com dores abdominais, melhorando com uso de anticonvulsivantes.

O mecanismo da EA segue baseada na existência de centros de controle das funções autônomas do rinencéfalo e córtex cerebral área 5, onde a estimulação anômala do giro hipocampal e do núcleo amigdaloide provoca alterações na função gastrointestinal gerando alguns sintomas de desconforto abdominal (RIBEIRO $\mathrm{RM}$ et al, 1964). Essas estruturas subcorticais são de extremo valor para o controle e manejo das manifestações vinculadas ao aparelho digestivo, principalmente por podermos associar com a clínica e com a eletroencefalografia apresentada em estudos e no presente relato.

As disfunções chamadas de disritmias cerebrais, apontadas pelo ECG, confirmam a presença de influência das estruturas subcorticais no aparelho digestivo. As disritmias cerebrais são uma alteração nas ondas elétricas cerebrais e seus ritmos próprios e são quase sempre associado as crises ou transtornos epiléticos, tecnicamente no cunho psiquiátrico não podem ser consideradas um patologia de sintomas únicos, mas sim uma gama de vários sintomas, diferentes entre si, que podem ser caracterizados por episódios periódicos ou seja paroxísticos e transitórios que alteram movimentos, estados de consciência e podem causar até mesmo as convulsões e, alterações de condutas e emoções (TREVISOL BPC, 1993).

A atividade epileptogênica dos lobos temporais e outros estímulos no córtex cerebral ativariam centros reguladores de tônus e atividade motora do trato gastrointestinal (DUTTA SR et al, 2007). Outro ponto a ser considerado no EEG de pacientes com epilepsia abdominal, é que existem geralmente específicas anormalidades no EEG, particularmente de um lobo temporal, além de transtorno convulsivo (ZARLING EJ, 1984) .O EEG representa então, um método bastante seguro para a identificação de diagnóstico diferencial entre uma síndrome clínica de caráter abdominal daquela decorrente de alguma patologia relacionada ao cérebro. No entanto, não é incomum para neurologistas pediátricos receber pacientes encaminhados com esta queixa. Portanto, é preciso estar ciente dos diagnósticos diferenciais desta condição, por exemplo considerando a enxaqueca como uma possibilidade porque é muito mais frequente que a epilepsia.

A fisiopatologia da EA permanece pouco conhecida nos dias atuais. Algumas etiologias foram consideradas, como prematuridade, convulsões febris e disfunção neuroendócrina, mas eles não são convincentes. Há um relato de um caso de dor abdominal e devido a um tumor cerebral-astrocitoma na área temporal. Mais recentemente, outro relato de caso mostrou malformação cortical, descrita como "Perisylvian bilateral polimicrogiria" na ressonância magnética associada a epilepsia porem sem muita certeza da fisiopatologia por detrás dessas patologias. Já no nosso relato não existem tais alterações por conta dessas patologias apresentadas, porem tanto essas doenças como o relato convergem para epilepsia abdominal em sua forma autêntica (FRANZON RC et al, 2002). Quando a dor abdominal apresenta como um curto episódio paroxístico seguido por qualquer distúrbio de consciência ou automatismos, pode ser facilmente siderada como uma crise epilética e, nesses casos, o lobo temporal é a origem mais provável da crise.

Uma resposta sustentada aos anticonvulsivantes tem sido aceita como um dos critérios para o diagnóstico de pacientes com epilepsia abdominal, em nosso caso a paciente foi tratada com oxicarbamazepina cujo mecanismo de ação baseia-se principalmente no bloqueio de canais de sódio voltagem-dependentes, resultando então na estabilização de membranas neurais hiperexcitadas (ARAÚJO DS, 2010), confirmados no eletroencefalograma realizado pela a paciente do caso (Figura 2), sendo assim responsável pela inibição da descarga neuronal repetitiva e diminuição da propagação de impulsos sinápticos, interrompendo as crises de epilepsia abdominal (ZINKIN NT, 2005). 


\section{CONSIDERAÇÕES FINAIS}

A epilepsia abdominal é considerada bastante rara e em alguns casos não muito bem diagnosticadas devido a uma gama de complexidade e raridades pertinentes a essa patologia. Deste modo essa análise visa demonstrar o quão raro a epilepsia abdominal se mostra e o quanto é importante ser realizado seu diagnóstico de forma clara, pautada em exames clínicos e complementares. Nesse paciente os sintomas foram bem controlados na média de 3 meses de acompanhamento e não houve efeitos adversos relatados. Sugerimos que em paciente com paroxismos de dor abdominal associado a sintomas gastrointestinais com ou sem manifestações do sistema nervoso, após a exclusão de etiologias mais comuns, a possibilidade da epilepsia abdominal deva ser considerada. Apesar de ser uma patologia pouco estudada é crucial analisar qualquer caso que se mostre em descobertas médicas. Deste modo, podemos classificar a paciente citada com diagnóstico de EA pela melhora total dos sintomas comprovadas após início do tratamento com oxicarbamazepina.

Deste modo podemos classificar a paciente citada em um exemplar de epilepsia abdominal com suas características comprovadas em anamnese, exame físico e exames laboratoriais e de imagem. Análise multidisciplinar é preciso entre os profissionais para que seja realizado o correto diagnóstico e tratamento. Existem poucos casos demonstrados em literatura como os já comparados acima, e é de extrema importância para comunidade médica, mais especificamente na comunidade pediátrica e para a comunidade em geral que pode ser atingida por essa patologia pediátrica complexa e rara. Portanto é de suma importância que se entenda a análise deste relato como um verdadeiro norteamento para essa afecção neurológica pediátrica rara e de alto grau de complexidade diagnostica.

\section{REFERÊNCIAS}

1. ARAÚJO SHRR, FREITAS EM. Revista Eletrônica de Farmácia Vol 7 (4), 30- 45, 2010.

2. BOYLE JT. Recurrent abdominal pains: an update. Pediatr Rev 1997; 18:310-20.

3. DUTTA SR et al. Abdominal epilepsy, an uncommon cause of recurrent abdominal pain: a brief report. Gut. 2007;56(3):439-411.

4. FRANZON RC et al. Recurrent abdominal pain: when an epileptic seizure should be suspected? Arq. NeuroPsiquiatr. 2002;60(3A):628-630.

5. RIBEIRO RM et al. Abdominal epilepsy in children. Arq. Neuro-Psiquiatr. 1964;22(1):44-50.

6. SOLANA LJ, ALARCÓN FO, et al. Abdominal epilepsy in an adult patient. Rev Gastroenterol Mex 1994;59297-300.

7. TREVISOL BPC et al. J. bras. psiquiatr; 10(42): 541-545, nov./dez. 1993.

8. ZARLING EJ. Abdominal epilepsy: an unusual cause of recurrent abdominal pain. Am J Gastroenterol 1984;79687688.

9. ZINKIN NT, PEPPERCORN MA. Abdominal epilepsy. Best Pract Res Clin Gastroenterol 2005;19263-274. 